\title{
High Prevalence of Dystonia in the Faroe Islands: A Population-Based Study
}

\author{
Elan D. Louis ${ }^{\mathrm{a}-\mathrm{c}}$ Eina H. Eliasen ${ }^{\mathrm{d}}$ Christine Y. Kim $^{\mathrm{a}, \mathrm{c}}$ Monica Ferrer ${ }^{\mathrm{a}}$ \\ Shahin Gaini ${ }^{\mathrm{e}-\mathrm{g}}$ Maria Skaalum Petersen ${ }^{\mathrm{d}} \mathrm{e}$ \\ ${ }^{a}$ Department of Neurology, Yale School of Medicine, Yale University, New Haven, CT, USA; ${ }^{b}$ Department of \\ Chronic Disease Epidemiology, Yale School of Public Health, Yale University, New Haven, CT, USA; 'Center for \\ Neuroepidemiology and Clinical Neurological Research, Yale School of Medicine, Yale University, New Haven, CT, \\ USA; ${ }^{d}$ Department of Occupational Medicine and Public Health, The Faroese Hospital System, Tórshavn, Faroe \\ Islands; ${ }^{e}$ Centre for Health Science, Faculty of Health Sciences, University of the Faroe Islands, Tórshavn, Faroe

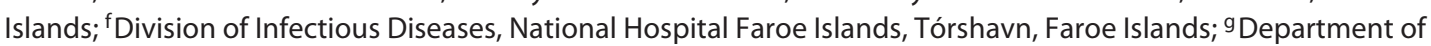 \\ Infectious Diseases, Odense University Hospital/University of Southern Denmark, Odense, Denmark
}

\section{Keywords}

Dystonia $\cdot$ Prevalence $\cdot$ Two-phase population-based design $\cdot$ Faroe Islands

\begin{abstract}
Background: There are fewer than 5 population-based studies of dystonia world wide. Only one utilized a movement disorders neurologist. Given the potential for founder effects, and the highly genetic nature of dystonia, the Faroe Islands provide a particularly interesting setting to study the prevalence of dystonia. Objective: To estimate the prevalence of dystonia. Methods: We used a 2-phase, population-based design, screening 1,334 randomly selected Faroese individuals aged $\geq 40$ years from which a subsample of 227 participated in an in-person clinical evaluation. Dystonia was assessed by 2 movement disorder neurologists using videotaped examinations. Results: Two of $227(0.88 \%$, 95\% $\mathrm{Cl}-0.33$ to $2.09 \%$ ) were diagnosed with cervical or segmental dystonia. An unusual form of thumb flexion dystonia was noted in 75 more, yielding a combined prevalence of $33.92 \%$ (95\% Cl 27.73-40.11\%). Conclusions: The prevalence of cervical or segmental dystonia was as high as in one prior population-based study using similar methods. Furthermore, an
\end{abstract}

unusual form of thumb flexion dystonia was uncovered, which yielded an extraordinarily high prevalence of dystonia in this population. Although our methods likely contributed to more complete capture of subtle dystonia, founder effects are highly likely to have been an additional major contributor to these findings.

(c) 2019 S. Karger AG, Basel

\section{Introduction}

Dystonia is a movement disorder characterized by sustained or intermittent muscle contractions causing abnormal, often repetitive, movements, postures, or both [1]. The postures or movements may be so mild that they are overlooked or they may be severe enough to produce significant disability [2]. The former scenario is quite common.

There are relatively few epidemiological studies of dystonia worldwide, and most have been service-based [3$5]$, with only 4 population-based studies [2, 6-8]. The population-based studies, from China [6], India [2], Egypt [7], and northern Italy [8], used a range of methodologies for ascertaining and diagnosing cases; 2 of these 
utilized movement disorder neurologists $[2,8]$, whereas 2 did not $[6,7]$. In the 2 that utilized a movement disorder neurologist, 1 employed the neurologist during an early phase of the study [8]; the other did so during a more terminal phase [2], thereby likely resulting in a lower estimate of dystonia prevalence. In these 2 studies, the prevalence of primary dystonia ranged from 44 per 10,000 [2] to 732 per 100,000 [8], demonstrating the more-than oneorder-of-magnitude difference between estimates that resulted from divergent methods.

The Faroe Islands are located in the North Atlantic Ocean, between Norway and Iceland and are inhabited by 49,121 individuals of whom 24,154 are age 40 or older (January 1, 2016). Due to the isolated geographic location and homogenous population [9], the Faroe Islands have been the focus of epidemiological investigations of a number of neurological disorders - the prevalence of Parkinson's disease (PD) $[10,11]$ is high, whereas essential tremor (ET) [12], multiple sclerosis [13, 14], and amyotrophic lateral sclerosis [15] are comparable to other European countries. Given the potential for founder effects in the Islands, and the highly genetic nature of dystonia [16], the Faroe Islands provide a particularly interesting setting in which to study the prevalence of dystonia.

There has been one prior study of the prevalence of dystonia, and more specifically primary focal dystonia, in the Faroe Islands [17]. The prevalence was 60.2 per 100,000 , and the prevalence of cervical dystonia, 47.8 per 100,000 , was higher than described in any prior survey [17]. However, the study was service-based rather than population-based [17] and was therefore likely to have under ascertained cases. Thus, our aim was to estimate the prevalence of dystonia in a population-based sample in the Faroe Islands. Establishing a precise national prevalence estimate is valuable as knowledge about prevalence is of crucial importance in informing national policy development as well as planning for health services. Moreover, subgroup differences in prevalence may offer early clues about the existence of environmental or underlying biological factors that could either be of etiologic or mechanistic importance. In addition, it is important for researchers to be able to confidently identify and count cases before they can study them further [18].

\section{Methods}

\section{Study Population and Sampling Frame}

We used a 2-phase, population-based study design. The primary aim of the parent study was to study tremor [12]. The study was undertaken between August 2016 and December 2017. From the 24,154 individuals aged $\geq 40$ years living in the Faroe Islands, a screening group comprised 3,000 Faroese individuals aged $\geq 40$ years was selected (Fig. 1 in Eliasen et al. [12]). Briefly, from the 24,154 individuals aged $\geq 40$ years living in the Faroe Islands, 4,798 were selected using the Faroese Population Registry [12]. These 4,798 were selected from the Registry based on 6 randomly selected birthdates (10th, 16th, 17th, 18th, 22th, and 30th) [12]. From these 4,798 , we selected into the screening group all individuals age 70 and older $(n=1,155)$ as well as 1,845 individuals selected by random sampling [12]. Therefore, the screening group comprised 3,000 Faroese individuals aged $\geq 40$ years [12].

All study procedures were approved by the Ethical review Committee of the Faroe Islands and by the Institutional Review Board at Yale University, with participation on a voluntary basis. Signed informed consent was obtained from each enrollee.

\section{First Screening Phase}

In the first screening phase (September 2016 - December 2016), these 3,000 individuals received an invitation letter to participate [12]. The letter included instructions on how to draw 2 Archimedes spirals with each hand and 4 blank sheets of paper [12]. A total of 1,334 (44.5\%) individuals returned the completed screening package (Fig. 1 in Eliasen et al. [12]). Tremor on each spiral was rated by a senior movement disorders neurologist (E.D.L.) who used an ordinal clinical rating scale (0-3.0), which included ratings of 0 (none), 0.5 (very mild), 1.0 (mild), 1.5 (mildto-moderate), 2.0 (moderate), and 3.0 (severe), as used in prior epidemiological studies [19]. Based on data from the questionnaire and spiral scores, the participants were stratified into 4 groups: (1) those with a high likelihood of having ET (e.g., spiral ratings $\geq 1.5$ on 1 or more dominant hand spirals or having been diagnosed previously with ET or having endorsed "head tremor" on the questionnaire), (2) those with an intermediate likelihood of having ET (e.g., both non-dominant hand spiral ratings $\geq 1.5$ ), (3) those with a low-intermediate likelihood of having ET (e.g., a single nondominant arm spiral rating $\geq 1.5$ ), and (4) those with a low likelihood of having ET (e.g., spiral ratings $\leq 1)$ [12, 20].

\section{Second Phase}

In the second phase, a subsample of 282 individuals who had returned the spirals and questionnaire was invited to participate in an in-person clinical evaluation at the Department of Occupational Medicine and Public Health or in their own homes. The overarching goals of the parent study were to (1) determine the prevalence of tremor (esp. ET) and (2) compare ET cases to controls in terms of environmental exposures [12]. To achieve these goals, we needed to enroll ET cases as well as controls [12]. We were concerned that the number of ET cases might be low [12]; therefore, to maximize enrollment of ET cases, we enrolled more individuals from groups 1 and 2 than from the other 2 groups because individuals in groups 1 and 2 had the highest likelihoods of having ET [12]. Thus, all of individuals in group 1 (i.e., high likelihood of ET) were selected and invited for an in-person assessment as well as a random selection of $80 \%$ in group 2 (i.e., intermediate likelihood of ET), 35\% in group 3, and 7\% in group 4 (low likelihood of having ET; Fig. 2 in Eliasen et al. [12]). The total number targeted was 250 [12]. We invited 282 individuals for an in-person clinical examination, expected that some would decline [12].

The in-person clinical evaluation was conducted by a trained nurse (E.H.E.) and included a demographic and clinical question- 
naire and a detailed videotaped neurological examination. The videotaped examination included (1) an ET-specific metric comprised of one test for postural tremor and 5 for kinetic tremor (e.g., pouring, drinking) performed with each arm (12 tests total, with total tremor score $=0-36$ ) [21], (2) the motor portion of the Unified PD Rating Scale [22] excluding an assessment of rigidity, and (3) an assessment of dystonia. The assessment of dystonia was performed through review of views of the face, neck, trunk, and extremities, while seated, standing, and walking (including turning); with posture (arms extended in front of body and in wing-beating position); and with multiple tests of action (finger taps, handopening/closure, finger-to-nose, pouring/drinking/lifting water with a spoon, and alternating toe-heel taps). Handwriting was videotaped and reviewed. Audio recordings of sustained phonation and speech were also reviewed to assess for dysphonia.

\section{Diagnosis of Dystonia and Other Neurological Conditions}

The diagnosis of dystonia was made using published diagnostic criteria (sustained or intermittent muscle contractions causing abnormal, often repetitive, movements, postures, or both) based on initial review of a senior movement disorder neurologist (E.D.L.). Those identified as having cervical or segmental dystonia were also independently reviewed by a second movement disorder neurologist (C.Y.K.) and final diagnosis assigned by consensus [1]. During video review of the motor examinations, a recurring phenomenon was noted during performance of hand-opening/closure as included in the Unified PD Rating Scale [22] motor assessment. This was a tendency to flex the thumb beneath (rather than over) the fingers during hand-opening/closure. This phenomenon was noted in a substantial number of participants, although the majority of participants performed hand-opening as expected, with the thumb flexed over the fingers. We sought to define the phenomenology of thumb flexion in this setting. Therefore, we selected a representative series of 4 patients demonstrating the phenomenon and invited an international panel of 4 dystonia experts from diverse training lineage to independently comment on the phenomenology. They were requested to opine on the thumb flexion phenomenology which might reflect dystonia and what other causes might be possible. All 4 experts opined that the thumb flexion was a form of focal dystonia [23].

ET diagnoses were assigned by E.D.L. by review of questionnaire data and the videotaped neurological examination and based on published diagnostic criteria [24]. The diagnosis of $P D$ was based on published criteria [25].

\section{Statistical Analyses}

Statistical analyses were performed using SPSS version 24.0. Chi-square and Student $t$ tests were used to assess differences across groups. CI was $95 \%$.

\section{Results}

A total of 227 participants aged $\geq 40$ years participated in the in-person clinical evaluation. Of these, $2(0.88 \%$, $95 \%$ CI -0.33 to $2.09 \%$ ) were diagnosed with dystonia (ages 67 and 70 years, both female, neither diagnosed previously, one with a family history of PD). One had cervi- cal dystonia and the other had segmental dystonia involving the cervical region and upper limb. Neither had ET. Neither had head tremor. Of the remaining 225 participants, 75 (33.33\%, 95\% CI 27.17-39.49\%) had thumb flexion dystonia (including 13 in the setting of ET; mean age $=58.7 \pm 11.0$ years, age range $=40-85$ years, 34 [45.3\%] female, 3 [4.0\%] previously diagnosed with dystonia, 12 [16.0\%] with a family history of PD and 12 [17.3\%] with a family history of tremor). Combining the 2 dystonia groups, 77 of 227 participants had dystonia (33.92\%, 95\% CI 27.73-40.11\%). These 77 did not differ from the remaining 150 participants in terms of gender, prior diagnosis of dystonia, family history of $\mathrm{PD}$, or family history of tremor, although they were on average 5.5 years younger (data not shown). No participants had PD.

We assessed whether the individuals in the 4 ET likelihood groups (Groups 1, 2, 3, or 4) differed with respect to the proportion with cervical or segmental dystonia or thumb flexion dystonia. The 4 groups did not differ significantly (prevalence of cervical or segmental dystonia or thumb flexion dystonia not in the setting of ET $=38.5 \%$ in Group 1, 36.9\% in Group 2, 40.9\% in Group 3, and $32.0 \%$ in Group 4, chi-square $=3.74, p=0.71$ ), suggesting that our preferential selection of individuals with higher likelihoods of tremor (Groups 1,2) did not result in a biased selection of more individuals with cervical or segmental dystonia or dystonic thumb flexion.

\section{Discussion}

There are relatively few epidemiological studies of dystonia worldwide, and most of these have been servicebased [3-5], with only 4 population-based studies [2, $6-8$ ]. Only 2 of the 4 population-based studies used a movement disorder neurologist $[2,8]$ although in only one was the movement disorder neurologist brought in during an early phase of the study [8]. Thus, our aim was to estimate the prevalence of dystonia in a populationbased sample of individuals; as in the earlier study [8], a movement disorder neurologist evaluated the patients.

A substantial number of participants exhibited a form of thumb-flexion dystonia during a rapid alternating movement task. This form of dystonia has not previously reported to our knowledge. An international panel of dystonia experts opined that the movement reflected dystonia, raising a thoughtful discussion about other possibilities but eventually independently and uniformly opining that this was a form of dystonia [23]. Had a detailed movement disorder examination not been performed 
and had the examination not been reviewed by a senior movement disorder neurologist, it is highly likely that this would not have been noted, and that our estimate of dystonia would have been very different. Furthermore, the particular hand-opening and closing task our subjects performed is not typically performed during prevalence studies of dystonia, as it is more often used to assess Parkinsonism. These observations reinforce the notion that issues of study design can greatly influence the sensitivity of one's evaluation. Indeed, it is not uncommon for dystonia to go unrecognized and undiagnosed. In family studies of dystonia, probands fail to report dystonia in most of their affected relatives - for example, in one study the sensitivity of the proband report was only $27.0 \%$ [26]. A study of patients with various forms of dystonia showed that in patients with hand dystonia, the latency from symptom onset to diagnosis was on average 10.1 years and in a substantial number of cases many years longer [27]. Diagnostic accuracy varies considerably among physicians; experienced neurologists are able to diagnose focal dystonia with greater accuracy than are general neurologists $[28,29]$.

Due to the isolated geographic location and homogenous population [9], the Faroe Islands have been the focus of epidemiological investigations of a number of neurological disorders. Given the potential for founder effects in the Islands, and the highly genetic nature of dystonia [16], the Faroe Islands provide a particularly interesting setting in which to study the prevalence of dystonia. Our study is most comparable to the populationbased study in northern Italy that utilized a movement disorders neurologist; in that study, the prevalence of primary dystonia was 732 per $100,000(0.732 \%)$ [8]. The prevalence of cervical or segmental dystonia in our sample was similar to that $(0.88 \%)$. However, when we include those with thumb flexion dystonia, our prevalence, $33.92 \%$, was extraordinarily high, likely for reasons discussed above (use of detailed videotaped examinations, review by movement disorder neurologist, founder effects). The prevalence was far higher than that reported in the prior service-based study in the Faroe Islands - 60.2 per 100,000 (0.0602\%) [17].

One issue is whether our method of ascertaining cases, who were enrolled in a study to identify tremor cases, resulted in an exaggerated prevalence of dystonia. That is, we stratified individuals based on likelihood of tremor and preferentially sampled those with higher likelihoods of tremor (Groups 1,2). Our additional analyses did not support the notion that this sampling scheme resulted in a higher number of dystonia cases.

Prevalence of Dystonia in the Faroe Islands
One other issue is that it is possible that our method of ascertaining cases (i.e., greater sampling of individuals with tremor) could have resulted in the pattern of dystonia we observed. One prior study showed that primary adult-onset dystonia cases with tremor are more likely to have cervical dystonia than blepharospasm [30]. Indeed, both of our cases of dystonia had cervical dystonia. It is important to note, however, that only one of our dystonia cases had tremor that was above normal levels and the other did not have tremor in the body region that had dystonic postures (i.e., the neck).

This study has several strengths. First, the study was population-based; as such, it is one of few such studies of the prevalence of dystonia [2,6-8]. Second, uniformity of examination across subjects ensured a consistent and thorough approach to the capture of clinical phenomenology. Third, the examination was extremely detailed, with assessments of a broad range movement disorders (tremor, dystonia, Parkinsonism). Fourth, all videotaped examinations were systematically quantified by a senior movement disorder neurologist. Fifth, the videotaping of the examination allowed for repeated review and clarification of subtle movement phenomenology. Finally, the study was one of only 2 such population-based studies of dystonia that have utilized a movement disorder neurologist in this manner [8].

There are also limitations. First, the sample size was small, with several 100 enrollees; hence, CIs were relatively wide. Second, the study was undertaken within the context of a very homogeneous population. Thus, the results of this work may not generalize to largely mixed populations. Third, we acknowledge that our method of ascertaining cases (i.e., greater sampling of individuals with tremor) could have resulted in the pattern of dystonia we observed. Finally, we did not query individuals about a family history of dystonia, so this information was not available.

In summary, in this population-based sample of individuals age 40 and older who were assessed using highly sensitive methods, the prevalence of cervical or segmental dystonia was as high as in one other population population-based study that used similar methods, indicating that dystonia is not as rare as previously considered. Furthermore, an unusual form of thumb flexion dystonia was also uncovered, which indicated an extraordinarily high prevalence of dystonia in this population. Although sensitive methods are likely to have contributed to more complete capture of even subtle forms of dystonia, founder effects are highly likely to have been a major contributor to our findings.

Neuroepidemiology 2019;53:220-224 223 


\section{Acknowledgments}

The authors thank the participants of the study for their support.

\section{Disclosure Statement}

There are no conflicts of interest or competing financial interests.

\section{Statement of Ethics}

All study procedures were approved by the ethical review committee of the Faroe Islands and by the Institutional Review Board at Yale University, with participation on a voluntary basis and signed informed consent obtained from each enrollee.

\section{Funding Sources}

This work was supported by NIH grants R01 NS039422 and NS094607 (Dr. Elan D. Louis).

\section{References}

1 Albanese A, Bhatia K, Bressman SB, Delong MR, Fahn S, Fung VS, et al. Phenomenology and classification of dystonia: a consensus update. Mov Disord. 2013 Jun;28(7):863-73.

2 Das SK, Banerjee TK, Biswas A, Roy T, Raut DK, Chaudhuri A, et al. Community survey of primary dystonia in the city of Kolkata, India. Mov Disord. 2007 Oct;22(14):2031-6.

3 Defazio G, Jankovic J, Giel JL, Papapetropoulos S: Descriptive epidemiology of cervical dystonia. Tremor Other Hyperkinet Mov (N Y). 2013 Nov;3. pii:tre-03-193-4374-2.

4 Defazio G. The epidemiology of primary dystonia: current evidence and perspectives. Eur J Neurol. 2010 Jul;17 Suppl 1:9-14.

5 Steeves TD, Day L, Dykeman J, Jette N, Pringsheim $\mathrm{T}$. The prevalence of primary dystonia: a systematic review and meta-analysis. Mov Disord. 2012 Dec;27(14):1789-96.

6 Li SC, Schoenberg BS, Wang CC, Cheng XM, Rui DY, Bolis CL, et al. A prevalence survey of Parkinson's disease and other movement disorders in the People's Republic of China. Arch Neurol. 1985 Jul;42(7):655-7.

7 Kandil MR, Tohamy SA, Fattah MA, Ahmed HN, Farwiez HM. Prevalence of chorea, dystonia and athetosis in Assiut, Egypt: a clinical and epidemiological study. Neuroepidemiology. 1994;13(5):202-10.

8 Müller J, Kiechl S, Wenning GK, Seppi K, Willeit J, Gasperi A, et al. The prevalence of primary dystonia in the general community. Neurology. 2002 Sep;59(6):941-3.

9 Als TD, Jorgensen TH, Børglum AD, Petersen PA, Mors O, Wang AG. Highly discrepant proportions of female and male Scandinavian and British Isles ancestry within the isolated population of the Faroe Islands. Eur J Hum Genet. 2006 Apr;14(4):497-504.

10 Petersen MS, Bech S, Nosova E, Aasly J, Farrer MJ. Familial aggregation of Parkinson's disease in the Faroe Islands. Mov Disord. 2015 Apr;30(4):538-44.

11 Petersen MS, Guella I, Bech S, Gustavsson E, Farrer MJ. Parkinson's disease, genetic variability and the Faroe Islands. Parkinsonism Relat Disord. 2015 Jan;21(1):75-8.
12 Eliasen EH, Ferrer M, Gaini S, Louis ED, Petersen MS. Prevalence of Essential Tremor in the Faroe Islands: A Population-Based Study. Neuroepidemiology. 2019;52(3-4):227-36.

13 Binzer S, Imrell K, Binzer M, Kyvik KO, Hillert J, Stenager E. High inbreeding in the Faroe Islands does not appear to constitute a risk factor for multiple sclerosis. Mult Scler. 2015 Jul;21(8):996-1002.

14 Joensen P. Multiple sclerosis: variation of incidence of onset over time in the Faroe Islands. Mult Scler. 2011 Feb;17(2):241-4.

15 Joensen P. Incidence of amyotrophic lateral sclerosis in the Faroe Islands. Acta Neurol Scand. 2012 Jul;126(1):62-6.

16 Lohmann K, Klein C. Update on the Genetics of Dystonia. Curr Neurol Neurosci Rep. 2017 Mar;17(3):26.

17 Joensen P. High prevalence of primary focal dystonia in the Faroe Islands. Acta Neurol Scand. 2016 Jan;133(1):55-60.

18 Louis ED, Ferreira JJ. How common is the most common adult movement disorder? Update on the worldwide prevalence of essential tremor. Mov Disord. 2010 Apr;25(5):53441.

19 Louis ED, Zhao Q, Meng H, Ding D: Screening for Action Tremor in Epidemiological Field Surveys: Assessing the Reliability of a Semi-Quantitative, Visual, Template-Based Scale for Rating Hand-Drawn Spirals. Tremor Other Hyperkinet Mov (N Y). 2012;2. pii:tre-02-46-177-2.

20 Louis ED, Ferrer M, Eliasen EH, Gaini S, Petersen MS. Tremor in normal adults: A population-based study of 1158 adults in the Faroe Islands. J Neurol Sci. 2019 May;400: 169-74.

21 Louis ED, Ottman R, Clark LN: Clinical classification of borderline cases in the family study of essential tremor: an analysis of phenotypic features. Tremor Other Hyperkinet Mov (N Y). 2014 Feb;4:220.

22 Goetz CG, Fahn S, Martinez-Martin P, Poewe W, Sampaio C, Stebbins GT, et al. Movement Disorder Society-sponsored revision of the Unified Parkinson's Disease Rating Scale
(MDS-UPDRS): Process, format, and clinimetric testing plan. Mov Disord. 2007 Jan; 22(1):41-7.

23 Kim CY, Eliasen EH, Skaalum Petersen M, Defazio G, Greene P, Jinnah HA, et al. Involuntary thumb flexion on neurological examination. An unusual form of upper limb dystonia in the Faroe Islands. Tremor Other Hyperkinet Mov (N Y). Forthcoming.

24 Louis ED, Ottman R, Ford B, Pullman S, Martinez $\mathrm{M}$, Fahn $\mathrm{S}$, et al. The Washington Heights-Inwood Genetic Study of Essential Tremor: methodologic issues in essentialtremor research. Neuroepidemiology. 1997; 16(3):124-33.

25 Hughes AJ, Ben-Shlomo Y, Daniel SE, Lees AJ. What features improve the accuracy of clinical diagnosis in Parkinson's disease: a clinicopathologic study. 1992. Neurology. 2001 Nov;57(10 Suppl 3):S34-8.

26 Martino D, Aniello MS, Masi G, Lamberti P, Lucchese V, Lamberti S, et al. Validity of family history data on primary adult-onset dystonia. Arch Neurol. 2004 Oct;61(10): 1569-73.

27 Macerollo A, Superbo M, Gigante AF, Livrea P, Defazio G. Diagnostic delay in adult-onset dystonia: data from an Italian movement disorder center. J Clin Neurosci. 2015 Mar;22(3): 608-10.

28 Defazio G, Albanese A, Pellicciari R, Scaglione CL, Esposito M, Morgante F, et al. Expert recommendations for diagnosing cervical, oromandibular, and limb dystonia. Neurol Sci. 2019 Jan;40(1):89-95.

29 Logroscino G, Livrea P, Anaclerio D, Aniello MS, Benedetto G, Cazzato G, et al. Agreement among neurologists on the clinical diagnosis of dystonia at different body sites. J Neurol Neurosurg Psychiatry. 2003 Mar;74(3):34850.

30 Defazio G, Gigante AF, Abbruzzese G, Bentivoglio AR, Colosimo C, Esposito M, et al. Tremor in primary adult-onset dystonia: prevalence and associated clinical features. J Neurol Neurosurg Psychiatry. 2013 Apr; 84(4):404-8. 